\title{
Plasma Energy Loss into Kaluza-Klein Modes
}

\author{
Duane A. Dicus ${ }^{1}$, Wayne W. Repko², and Vigdor L. Teplitz ${ }^{3,4}$ \\ ${ }^{1}$ Center for Particle Physics, University of Texas, Austin, TX 78712 \\ ${ }^{2}$ Department of Physics and Astronomy, Michigan State University, East Lansing, MI 48824 \\ ${ }^{3}$ Department of Physics, Southern Methodist University, Dallas, TX 75275. \\ ${ }^{4}$ Office of Science and Technology Policy, Executive Office of the President, Washington, DC 20001*
}

October 22,2018

\begin{abstract}
Recently, Barger et al. computed energy losses into Kaluza Klein modes from astrophysical plasmas in the approximation of zero density for the plasmas. We extend their work by considering the effects of finite density for two plasmon processes. Our results show that, for fixed temperature, the energy loss rate per $\mathrm{cm}^{3}$ is constant up to some critical density and then falls exponentially. This is true for transverse and longitudinal plasmons in both the direct and crossed channels over a wide range of temperature and density. A difficulty in deriving the appropriate covariant interaction energy at finite density and temperature is addressed. We find that, for the cases considered by Barger et al., the zero density approximation and the neglect of other plasmon processes is justified to better than an order of magnitude.
\end{abstract}

\section{Introduction}

Recently, Barger et al. 1 addressed astophysical constraints on extra dimensions by computing energy loss rates from the sun, red giant stars and (Type II) supernovae due to possible excitation of graviton modes, $\mathcal{G}$, in the case that the extra dimensions are compactified [2]. The processes $\gamma \gamma \rightarrow \mathcal{G}, e^{+} e^{-} \rightarrow \mathcal{G}, \gamma e \rightarrow \mathcal{G}, e N \rightarrow \mathcal{G} e N$ (in the static nucleon approximation), and $N N \rightarrow \mathcal{G} N N$ where considered. They worked in the zero density approximation, varying only the temperature. Their calculation neglected plasma effects and they anticipated that this neglect should not be important because of the high power dependence on $M_{S}$, the inverse of the compactification dimension.

The purpose of this paper is to address the extent to which the process $\gamma \gamma \rightarrow \mathcal{G}$ (and the crossed process $\gamma \rightarrow \gamma \mathcal{G}$ ) is affected by a non-zero charged particle density and the presence of both longitudinal and transverse plasmons. Our aims are, in brief, to find numerical

*Address until June 30, 2001 
values for the energy loss rate per $\mathrm{cm}^{3}$ for densities from 1 to $10^{15} \mathrm{gm} / \mathrm{cm}^{3}$ and temperatures from 1 to $10^{9} \mathrm{eV}$, to confirm the Barger et al. expectation of density insensitivity, to show the extent to which the systems they considered are close to the border in density at which their expectation fails, to determine the relative contributions of longitudinal and transverse plasmon processes, to address the size of the contribution of the crossed process, and, importantly, to note the ambiguities in the form of a covariant interaction between plasmons and Kaluza-Klein modes.

It is clear that the expectation of Barger et al. [1] must fail at sufficiently high density, for fixed temperature, since the energy loss rate goes as an integral over the Bose-Einstein distribution, $\left(e^{\omega_{i} / k T}-1\right)^{-1}$, in which the frequencies are given by a dispersion relation with an effective photon mass that grows with density. It is essentially a numerical question as to the point at which suppression sets in and the rate (in density) at which it proceeds. Intuition is hampered by the fact that the natural parameter is the electron chemical potential which is not simply related to the particle density. We give the numerical results over the temperature and density ranges cited above.

An important problem in carrying out this work is the question of the appropriate Lagrangian. The free space coupling between the electromagnetic field and gravitons can be found in textbooks, for example [3], and has been generalized to the case of higher dimensional Kaluza-Klein excitations [4, 5]. However, we have not found a parallel literature for the case in which the free space photon is replaced by a plasmon satisfying a non-trivial dispersion relation. This difficulty is addressed in Section 2. We adopt a diagrammatic approach and also make an approximation that we test numerically. Also given in Section 2 is the formalism used for the numerical calculations of Section 3. We conclude in Section 4 with a brief summary.

\section{Formalism}

In a medium with nonzero temperature and density, radiation satisfies the dispersion relation

$$
\omega^{2}-|\vec{k}|^{2}=\Pi_{a}(\omega,|\vec{k}|) .
$$

$\Pi_{a}(\omega,|\vec{k}|)$ is the transverse or longitudinal component of the polarization tensor,

$$
\Pi_{a}(\omega,|\vec{k}|)=\varepsilon_{a}^{\mu *} \Pi_{\mu \nu} \varepsilon_{a}^{\nu},
$$

where $\varepsilon_{a}^{\mu}(a=L, T)$ are the polarization vectors and the polarization tensor $\Pi_{\mu \nu}$ is the photon self energy in the medium. The contribution to this self energy from fermions in the medium is calculated in the medium rest frame by adding a term

$$
\frac{\not p+m}{e^{(E \pm \mu) / T}+1} 2 \pi i \delta\left(p^{2}-m^{2}\right)
$$

to the usual (vacuum) progagator. Here, $T$ is the temperature, $E=p^{0}$ and $\mu$ is the electron chemical potential, which is related to the electron number density $n_{e}$ by

$$
2 \int \frac{d^{3} p}{(2 \pi)^{3}}\left[\frac{1}{e^{(E-\mu) / T}+1}-\frac{1}{e^{(E+\mu) / T}+1}\right]=n_{e}
$$


To lowest order in the fine structure constant $\alpha$, the polarization tensor is given by

$$
\begin{aligned}
\Pi^{\mu \nu}=16 \pi & \alpha \int \frac{d^{3} p}{(2 \pi)^{3} 2 E}\left[\frac{1}{e^{(E-\mu) / T}+1}+\frac{1}{e^{(E+\mu) / T}+1}\right] \\
& \times \frac{(p \cdot k)^{2} g^{\mu \nu}+k^{2} p^{\mu} p^{\nu}-p \cdot k\left(k^{\mu} p^{\nu}+k^{\nu} p^{\mu}\right)}{(p \cdot k)^{2}} .
\end{aligned}
$$

It turns out that $\Pi_{L}$ and $\Pi_{T}$ can be approximated to within $1 \%$ for all temperatures and densities [6] by

$$
\begin{aligned}
& \Pi_{L}=\omega_{P}^{2}\left[1-G\left(v_{*}^{2}|\vec{k}|^{2} / \omega^{2}\right)\right]+v_{*}^{2}|\vec{k}|^{2}-|\vec{k}|^{2}, \\
& \Pi_{T}=\omega_{P}^{2}\left[1+\frac{1}{2} G\left(v_{*}^{2}|\vec{k}|^{2} / \omega^{2}\right)\right]
\end{aligned}
$$

where $v_{*}$ is an average value of $v=|\vec{p}| / E$ for the electron (the only fermion which contributes for stellar temperatures and densities). Explicitly,

$$
v_{*}=\omega_{1} / \omega_{P},
$$

with $\omega_{1}$ given by

$$
\omega_{1}^{2}=\frac{4 \alpha}{\pi} \int_{0}^{\infty} d|\vec{p}||\vec{p}|\left(\frac{5}{3} v^{3}-v^{5}\right) f_{E}
$$

where $f_{E}$ is the sum of the electron and positron distributions (the square bracket in Eq. (5) above). The plasma frequency $\omega_{P}$ is given by

$$
\omega_{P}^{2}=\frac{4 \alpha}{\pi} \int_{0}^{\infty} d|\vec{p}||\vec{p}|\left(v-\frac{1}{3} v^{3}\right) f_{E}
$$

and the function $G(x)$ is

$$
G(x)=\frac{3}{x}\left[1-\frac{2 x}{3}-\frac{1-x}{2 \sqrt{x}} \log \left(\frac{1+\sqrt{x}}{1-\sqrt{x}}\right)\right] .
$$

It will be important below to note that, for transverse photons, $k^{2}=\omega^{2}-|\vec{k}|^{2}$ is $\omega_{P}^{2}$ for $|\vec{k}|=0$ and increases as $|\vec{k}|$ increases, while, for longitudinal photons, $k^{2}=\omega_{P}^{2}$ at $|\vec{k}|=0$ and decreases as $|\vec{k}|$ increases. Integration over $|\vec{k}|$ for longitudinal photons must be cut off at the point where $k^{2}$ becomes negative,

$$
|\vec{k}|_{\max }^{2}=\frac{3 \omega_{P}^{2}}{v_{*}^{2}}\left[\frac{1}{2 v_{*}^{2}} \log \left(\frac{1+v_{*}}{1-v_{*}}\right)\right] .
$$

We include in our numerical evaluations the renormalization constants

$$
Z_{a}^{-1}=1-\frac{\partial \Pi_{a}}{\partial \omega^{2}}
$$


although this is inconsistent with calculating only to the lowest order in $\alpha$. It is a check on our results that they do not change significantly when the $Z_{a}$ are set to unity. The $Z_{a}$ are given by 7

$$
\begin{aligned}
Z_{T} & =\frac{2 \omega^{2}\left(\omega^{2}-v_{*}^{2}|\vec{k}|^{2}\right)}{\omega^{2}\left[2 \omega_{P}^{2}-2\left(\omega^{2}-|\vec{k}|^{2}\right)\right]+\left(\omega^{2}+|\vec{k}|^{2}\right)\left(\omega^{2}-v_{*}^{2}|\vec{k}|^{2}\right)} \\
Z_{L} & =\frac{2 \omega^{2}\left(\omega^{2}-v_{*}^{2}|\vec{k}|^{2}\right)}{3 \omega_{P}^{2}-\omega^{2}+v_{*}^{2}|\vec{k}|^{2}} \frac{\omega^{2}}{\omega^{2}-|\vec{k}|^{2}} .
\end{aligned}
$$

The rate of graviton emission can be calculated using the Lagrangian for the coupling of Kaluza-Klein field $\mathcal{G}_{\vec{n}}^{\mu \nu}$, corresponding to the mass excitation $m_{\vec{n}}^{2}=(2 \pi)^{2} \vec{n}^{2} / R^{2}$, to the photon energy-momentum tensor $T_{\mu \nu}$. Neglecting gauge terms, this coupling is [4, 5]

$$
\begin{aligned}
\mathcal{L} & =-\frac{\kappa}{2} \mathcal{G}_{\vec{n}}^{\mu \nu} T_{\mu \nu} \\
& =\frac{\kappa}{2}\left(\mathcal{G}_{\vec{n}}^{\mu \nu} F_{\mu}^{\lambda} F_{\nu \lambda}-\frac{1}{4} \mathcal{G}_{\vec{n}, \mu}^{\mu} F^{\lambda \rho} F_{\lambda \rho}\right),
\end{aligned}
$$

where $F_{\mu \nu}$ is the electromagnetic field tensor. We consider only the coupling of the spin-2 component of the Kaluza-Klein field; the spin- 0 component does not couple to photons.

The matrix element for $\gamma\left(k_{1}\right) \gamma\left(k_{2}\right) \rightarrow \mathcal{G}$ obtained from Eq. (16) is [4, [5]

$$
\mathcal{M}=\frac{\kappa}{2} \varepsilon^{\lambda}\left(k_{1}\right) \varepsilon^{\rho}\left(k_{2}\right) \varepsilon^{* \mu \nu}\left(k_{1}+k_{2}\right) T_{\mu \nu, \lambda \rho}^{(0)},
$$

where

$$
T_{\mu \nu, \lambda \rho}^{(0)}=k_{1} \cdot k_{2} C_{\mu \nu, \lambda \rho}+D_{\mu \nu, \lambda \rho}\left(k_{1}, k_{2}\right),
$$

with

$$
\begin{aligned}
C_{\mu \nu, \lambda \rho} & =\eta_{\mu \lambda} \eta_{\nu \rho}+\eta_{\mu \rho} \eta_{\nu \lambda}-\eta_{\mu \nu} \eta_{\lambda \rho} \\
D_{\mu \nu, \lambda \rho}\left(k_{1}, k_{2}\right) & =\eta_{\mu \nu} k_{1 \rho} k_{2 \lambda}-\left[\eta_{\mu \rho} k_{1 \nu} k_{2 \lambda}+\eta_{\mu \lambda} k_{1 \lambda} k_{2 \nu}+(\mu \leftrightarrow \nu)\right] .
\end{aligned}
$$

The sum over polarizations of the Kaluza-Klein state is [4, 5]

$$
\sum_{s=1}^{5} \varepsilon_{\mu \nu}^{s}(k) \varepsilon_{\lambda \rho}^{s *}(k)=\frac{1}{2} B_{\mu \nu, \lambda \rho}(k),
$$

with

$$
\begin{aligned}
B_{\mu \nu, \lambda \rho}(k) & =E_{\mu \lambda} E_{\nu \rho}+E_{\mu \rho} E_{\nu \lambda}-\frac{2}{3} E_{\mu \nu} E_{\lambda \rho} \\
E_{\mu \nu} & =\eta_{\mu \nu}-\frac{k_{\mu} k_{\nu}}{m_{\vec{n}}^{2}} .
\end{aligned}
$$


The coupling Eq. (18) is gauge invariant even if $k_{1}^{2}$ and $k_{2}^{2}$ are not zero, e.g. $k_{1}^{\mu} T_{\mu \nu, \lambda \rho}^{(0)}=0$. However, it is not conserved, $\left(k_{1}+k_{2}\right)^{\mu} T_{\mu \nu, \lambda \rho}^{(0)} \neq 0$, if $k_{1}^{2}$ and/or $k_{2}^{2}$ differs from zero. We cannot write a conserved coupling by using the energy-momentum tensor for a massive vector field because $k_{1}^{2}$ is not necessarily equal to $k_{2}^{2}$. This means that if we square $\mathcal{M}$ of Eq. (17), and use Eq. (21), we get extra terms of the form $k_{1}^{2} / m_{\vec{n}}^{2}$ or $k_{2}^{2} / m_{\vec{n}}^{2}$ from the second term in Eq. (23).

To have a conserved amplitude with $k_{1}^{2} \neq k_{2}^{2} \neq 0$, we must include all the diagrams of Fig. (11). The Feynman rules for the fermion-fermion- $\mathcal{G}$ coupling and the fermion-fermion-

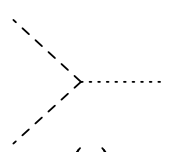

(a)

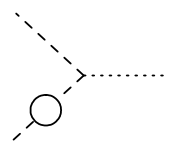

(b)
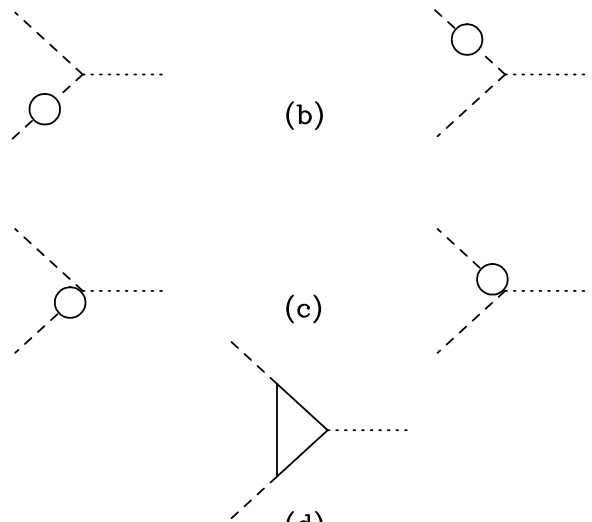

(d)

Figure 1: The Feynman diagrams for the one-loop corrections to the energy-momentum tensor are shown. The dotted lines denote Kaluza-Klein gravitons, the dashed lines photons and the solid lines electrons.

photon- $\mathcal{G}$ are given in Refs. 4, 5], and the loops are calculated by using Eq. (3) for one of the legs. We have shown the the sum of these diagrams is gauge invariant and conserved for arbitrary $k_{1}^{2}$ and $k_{2}^{2}$ at finite temperature and density. However, this was done without actually evaluating the diagrams. In particular, diagram (d) is very tedious and we have not computed it. Instead, we have used only diagram (a) (Eq. (17) above) but have evaluated every energy loss twice - once including the $k_{1}^{2} / m_{\vec{n}}^{2}$ and $k_{2}^{2} / m_{\vec{n}}^{2}$ terms and once omitting them. In every case, the results were almost identical. While this proves nothing, it does seem to indicate that performing the complete one-loop calculation would not give a substantially different answer.

The reaction rate must be summed over the Kaluza-Klein states, which is done by integrating over

$$
d m_{n}^{2} \frac{4 \pi m_{n}^{(n-2)}}{\kappa^{2} M_{S}^{n+2}}
$$

where $n$ is the number of extra dimensions. $M_{S}$ is the string scale which is related to the 
compactification scale $R$ and Newton's constant $G_{N}$. Specifically, we use

$$
M_{S}^{n+2} R^{n}=\frac{(4 \pi)^{n / 2} \Gamma(n / 2)}{4 G_{N}} .
$$

Our definition of $M_{S}^{n+2}$ differs from that of [1] by a factor of 2, i.e. their $M_{S}$ is larger by a factor $2^{1 /(n+2)}$. As a consequence, values of the energy loss per unit volume obtained from our tables must be multiplied by 2 when comparing with Barger et al. [1].

For 2 particles $\rightarrow 1$ particle reactions there remains a delta function from phase space which identifies $m_{n}^{2}$ with the center of mass squared energy $s$. Thus, the integral over $m_{n}^{2}$, Eq. (24), replaces $m_{n}^{2}$ by $s$ and our results depend on $n$ through the factor $s^{(n-2) / 2} / M_{S}^{(n+2)}$.

The rate of energy loss per unit volume is given by the standard expression

$$
\mathcal{Q}_{a, b}=A \int \frac{d^{3} k_{1}}{(2 \pi)^{3}} \frac{1}{e^{\omega_{1} / T}-1} \int \frac{d^{3} k_{2}}{(2 \pi)^{3}} \frac{1}{e^{\omega_{2} / T}-1}\left(\omega_{1}+\omega_{2}\right) Z_{a}\left(\left|\vec{k}_{1}\right|\right) Z_{b}\left(\left|\vec{k}_{2}\right|\right) v \sigma
$$

where $v \sigma$ denotes the cross section times the relative velocity [1]. The initial photons have $k_{i}^{2}=\omega_{i}^{2}-\left|\vec{k}_{i}\right|^{2}, i=1,2$ and can be transverse, $a=b=T$, longitudinal, $a=b=L$ or mixed, e.g. $a=L, b=T$. The factor $A$ gives the number of spin states: $A=4,2,1$ for $T T, T L$ or

$L L$. For longitudinal photons, the $|\vec{k}|$ integrals are cut off at $|\vec{k}|_{\max }$ given by Eq. (12). The corresponding expression for the energy loss in the decay $T \rightarrow L \mathcal{G}$ is

$$
\begin{aligned}
\mathcal{Q}_{T \rightarrow L}=2 \int \frac{d^{3} k_{T}}{(2 \pi)^{3} 2 \omega_{T}} \frac{1}{e^{\omega_{T} / T}-1} \int \frac{d^{3} k_{L}}{(2 \pi)^{3} 2 \omega_{L}} \frac{\left(\omega_{T}-\omega_{L}\right)}{1-e^{-\omega_{L} / T}} Z_{T}\left(\left|\vec{k}_{T}\right|\right) Z_{L}\left(\left|\vec{k}_{L}\right|\right) \\
\times \frac{(2 \pi)^{4}\left(\left(k_{T}-k_{L}\right)^{2}\right)^{(n-2) / 2}}{\kappa^{2} M_{S}^{n+2}}|\mathcal{M}|^{2},
\end{aligned}
$$

where $\mathcal{M}$ is given by Eq. (17) with $k_{2} \rightarrow-k_{L}$.

\section{Calculations}

The first step in the calculations is to obtain $\mu(T, \rho)$ from Eq. (4). In doing this we assume that the electron number density, $n_{e}$ is related to the mass density $\rho$ by $n_{e}=\rho / m_{p}$ where $m_{p}$ is the proton mass. This is useful for comparison purposes and is a reasonable order of magnitude approximation but needs correction (by less than an order of magnitude) for a supernova or a neutron star. The results of the calculation of $\mu$ are given in Tables [10 and [1] for the matrix of $\rho$ and $T$ values: $\rho=1.0,10.0, \ldots 10^{15} \mathrm{gm} / \mathrm{cm}^{3}$ and $T=1,10^{2}, \ldots 10^{9} \mathrm{eV}$. Two tables are given $\left(\mu(T, \rho)\right.$ and $\left.\tilde{\mu}(T, \rho)=\mu(T, \rho)-m_{e}\right)$ in order to make clear both the deviation of $\mu$ from $m_{e}$ (taken as $0.51 \mathrm{MeV}$ ) at low temperature and its deviation from zero at high. Note the rapid variation of $\mu$ (for the lowest densities) at the temperature $T$ around $0.1 \mathrm{MeV}$ where pair production first begins to be copious, and the slower but similar variation at higher densities $\rho$. The variation is slower for higher densities because the electron-positron density difference needs to have a large value. These variations are 


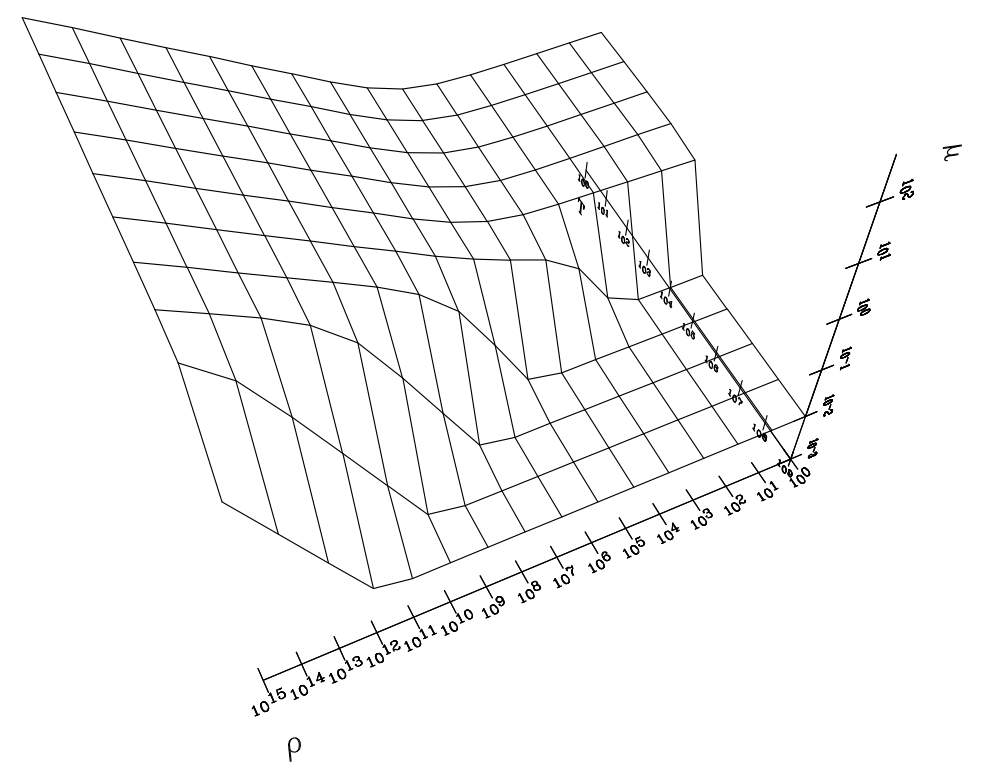

Figure 2: The chemical potential $\mu(\rho, T)$ is plotted for the range of density $\rho$ and temperature $T$ given in the text. Here, for display purposes, the lowest value of $\mu$ shown, $10^{-2}$, is an upper limit on the exact numbers in Table 10.

illustrated in Fig.2] where, for display purposes, the lowest value of $\mu$ shown, $10^{-2}$, is an upper limit on the exact numbers in Table 10. In calculating $\mu$ we used an iteration procedure and required that the output density value equal the input to better than a percent.

We now pass on to the results of calculating $\mathcal{Q}=d^{4} E / d t d V$ for the case of $n=2$ extra dimensions. It was possible to evaluate the integral in Eq. (26) over the cosine of the angle between the two plasmons analytically so that, for all the processes under consideration, only two integrals remain in finding the energy loss rate - the integrals over the two plasmon momenta.

It should be noted that there are only four processes to consider: (1) $T+T \rightarrow \mathcal{G}$, (2) $T+L \rightarrow \mathcal{G}$, (3) $T \rightarrow L+\mathcal{G}$, and (4) $L+L \rightarrow \mathcal{G}$. This is because, as the plasmon momentum $|\vec{k}|$ increases, the effective mass of a transverse plasmon increases while the effective mass of a longitudinal plasmon decreases [7]. Thus the missing processes, $L \rightarrow T+\mathcal{G}$ and $L \rightarrow L+\mathcal{G}$ are forbidden by energy-momentum conservation. The assertion is clear for the first process since $m_{T}>m_{L}$. For the second, we note that, in the rest frame of the decaying longitudinal plasmon, $m_{L}=\omega_{P}$ and conservation of energy and momentum implies that the graviton mass, $m_{\mathcal{G}}$, satisfies

$$
m_{\mathcal{G}}^{2}=\left(\omega_{P}-\omega_{L}(|\vec{k}|)\right)^{2}-|\vec{k}|^{2},
$$

where $\omega_{L}(|\vec{k}|)$ is the energy of the final plasmon. Using the dispersion relation for longitudinal plasmons, it can be shown that the right side of Eq. (28) is less than zero for $|\vec{k}|>0$.

The results of the calculations are given in Tables 105. These have the energy loss rates for the four processes, and for the sum, for a matrix of density and temperature values - 1.0 to $10^{15} \mathrm{gm} / \mathrm{cm}^{3}$ for density $\rho$ and 1 to $10^{9} \mathrm{eV}$ for temperature $T$ - in both cases in factors 
of 10 increments. In these tables, $T$ increases from left to right while $\rho$ increases from top to bottom. The entries are logs to the base 10 of the energy loss rate in ergs per $\mathrm{cm}^{3}$-s. Note that Barger et al. 1] give results per unit mass, but results per unit volume are better for our purposes since they show more clearly the way in which the zero-density approximation breaks down as the density increases. We give the results for $M_{S}=1 \mathrm{TeV}$. Two additional tables, 6] and [7] give respectively the number of the process that dominates for the reaction (zero if the rate is zero, i.e. below $10^{-320}$ ) and the fraction of the total represented by the dominant contribution.

In Fig. $3(T+T \rightarrow \mathcal{G})$, we see at a glance the effect cited in the Introduction: for fixed $T$ the

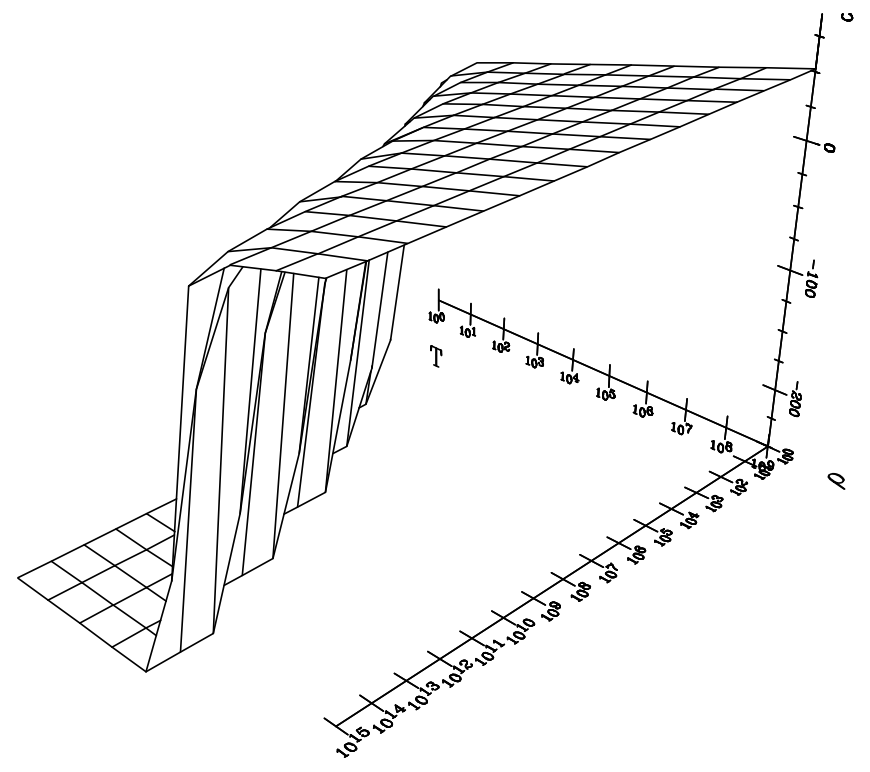

Figure 3: The $\log _{10}(\mathcal{Q})$ for the process $T+T \rightarrow \mathcal{G}$ is plotted for the range of density $\rho$ and temperature $T$ given in the text.

energy loss rate is independent of the density until $\rho$ increases to a point where the effective photon mass and plasmon density are sufficiently high that the rate drops exponentially. The numerical values are given in Table1. The analogous plot for $L+L \rightarrow \mathcal{G}$ is shown in Fig.4. Here, the the rate grows slightly for a fixed $T$ before dropping exponentially in the mass of the longitudinal plasmon with increasing $|\vec{k}|$. Again, numerical values are found in Table 4 .

The Sun, a red giant, and a Type II supernova are, in the $(\rho, T)$ plane, given by Barger et al. [1] to be at $\left(156 \mathrm{gm} / \mathrm{cm}^{3}, 1.3 \mathrm{keV}\right),\left(10^{6} \mathrm{gm} / \mathrm{cm}^{3}, 8.6 \mathrm{keV}\right)$, and $\left(10^{15} \mathrm{gm} / \mathrm{cm}^{3}, 30\right.$ $\mathrm{MeV}$ ) respectively. We see from Table 1 that the Sun is in a low density region where the zero-density approximation holds while the supernova is on the edge of a constant density region. We also see from Table 6 that the other processes contribute little in these two cases. The red giant (RG) case is more interesting. In Table 1, we see from the $T=10^{4}$ and $T=10^{3}$ columns that the $\mathrm{RG}$ is in the gentle fall off region for the former, but the steep fall off region for the latter. Examining the region between 1 and $10 \mathrm{keV}$ more closely gives, with $\mathrm{T}$ varying in $1.0 \mathrm{keV}$ increments, for the log of the TT energy loss rate: -16.7, -5.1 , 


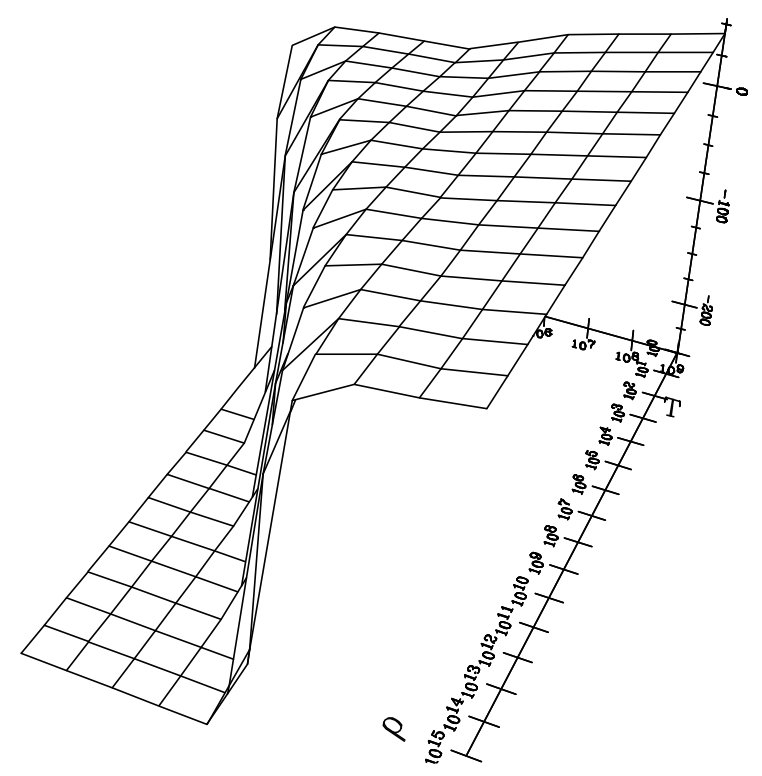

Figure 4: The $\log _{10}(\mathcal{Q})$ for the process $L+L \rightarrow \mathcal{G}$ is plotted for the range of density $\rho$ and temperature $T$ given in the text.

$-0.91,1.36,2.85,3.94,4.78,5.48,6.07,6.57$. The total energy loss rate varies as: -15.96 , $-4.14,0.37,2.65,3.77,4.59,5.25,5.82,6.31,6.77$. In short, at the RG, the TT energy loss rate, $1.4 \mathrm{erg} / \mathrm{g} \cdot \mathrm{s}$, is down by a factor $4.5 \mathrm{from}$ its zero-density approximation [1, but the other processes bring the total rate up to $2.6 \mathrm{erg} / \mathrm{g} \cdot \mathrm{s}$, or within about a factor 2 of the zero density result.

Finally we turn to the dependence of the emission rates on the number $n$ of (large) extra dimensions. We give, in Tables 8 and 9 , the results for the total rates for $n=3$ and $n=4$. One sees, in the low-density limit, the $T^{7+n}$ behavior pointed out by Barger et al. 1] for the $T T \rightarrow \mathcal{G}$ rate which dominates for $n=3,4$ in the same places as in the $n=2$ case.

\section{Summary}

The approximations of zero density and purely transverse $(T+T \rightarrow \mathcal{G})$ photon annihilation into gravitons of [1] must fail, for fixed temperature, at high densities. We have computed a first estimate of finite density corrections to two-plasmon production, for both transverse and longitudinal plasmons of Kaluza-Klein excitations, as well as the decay process $T \rightarrow L+\mathcal{G}$ as a function of plasma density and temperature over a wide range of interest in both parameters. Our conclusion is that the zero-density, pure transverse approximation is satisfactory for the sun, marginal for supernovae, and fails by about a factor of 5 for red giants. It is interesting to note that, while very little of the $\rho-T$ plane is occupied, astrophysical systems appear to be preferentially located relatively close to the boundaries (in $\rho$ ) at which the transverse photon approximation begins to fail. Our calculation is approximate in that we omit most of the diagrams of Fig. (11). However, we believe our results indicate that the full calculation of all the diagrams is unlikely to modify our conclusions. 


\section{Acknowledgements}

One of us (V. T.) wishes to thank R. Mohapatra for helpful conversations. This work was supported in part by the National Science Foundation under grant PHY-9802439 and by the Department of Energy under Contract Nos. DE-FG03-93ER40757 and DE-FG0395ER40908. 


\section{References}

[1] V. Barger, T. Han, C. Kao and R.-J. Zhang, Phys. Lett. B461, 34 (1999); hep-ph/9905474 (1999).

[2] N. Arkani-Hamed, S. Dimopoulos and G. Dvali, Phys. Lett. B 429, 263 (1998); I. Antoniadis, N. Arkani-Hamed, S. Dimopoulos and G. Dvali ibid., 436, 506 (1998).

[3] S. Weinberg, Gravitation and Cosmology, John Wiley and Sons, New York, 1972.

[4] G. F. Guidice, R. Ratazzi and J. D. Wells, Nucl. Phys. B 544, 3 (1999).

[5] T. Han, J. D. Lykken and R.-J. Zhang, Phys. Rev. D 59, 105006 (1999).

[6] E. Bratten and D. Segel, Phys. Rev. D 48, 1478 (1993).

[7] Georg G. Raffelt, Stars as Laboratories for Fundamental Physics, The University of Chicago Press, 1996. 


\section{Tables}

\begin{tabular}{|c|c|c|c|c|c|c|c|c|c|c|}
\hline$\rho \backslash T$ & 1 & $10^{1}$ & $10^{2}$ & $10^{3}$ & $10^{4}$ & $10^{5}$ & $10^{6}$ & $10^{7}$ & $10^{8}$ & $10^{9}$ \\
\hline 1 & -47.34 & -20.54 & -10.89 & -1.88 & 7.12 & 16.12 & 25.12 & 34.12 & 43.12 & 52.12 \\
\hline $10^{1}$ & -99.18 & -23.76 & -10.97 & -1.88 & 7.12 & 16.12 & 25.12 & 34.12 & 43.12 & 52.12 \\
\hline $10^{2}$ & -267.65 & -38.33 & -11.54 & -1.89 & 7.12 & 16.12 & 25.12 & 34.12 & 43.12 & 52.12 \\
\hline $10^{3}$ & & -89.99 & -14.75 & -1.97 & 7.12 & 16.12 & 25.12 & 34.12 & 43.12 & 52.12 \\
\hline $10^{4}$ & & -255.96 & -29.09 & -2.53 & 7.11 & 16.12 & 25.12 & 34.12 & 43.12 & 52.12 \\
\hline $10^{5}$ & & & -77.48 & -5.52 & 7.03 & 16.12 & 25.12 & 34.12 & 43.12 & 52.12 \\
\hline $10^{6}$ & & & -210.07 & -16.77 & 6.57 & 16.11 & 25.12 & 34.12 & 43.12 & 52.12 \\
\hline $10^{7}$ & & & & -45.16 & 4.96 & 16.06 & 25.12 & 34.12 & 43.12 & 52.12 \\
\hline $10^{8}$ & & & & -106.83 & 0.36 & 15.86 & 25.12 & 34.12 & 43.12 & 52.12 \\
\hline $10^{9}$ & & & & -239.38 & -11.34 & 15.32 & 25.10 & 34.12 & 43.12 & 52.12 \\
\hline $10^{10}$ & & & & & -38.58 & 13.77 & 25.03 & 34.12 & 43.12 & 52.12 \\
\hline $10^{11}$ & & & & & -99.08 & 9.25 & 24.84 & 34.12 & 43.12 & 52.12 \\
\hline $10^{12}$ & & & & & -230.97 & -2.40 & 24.32 & 34.10 & 43.12 & 52.12 \\
\hline $10^{13}$ & & & & & & -29.61 & 22.77 & 34.03 & 43.12 & 52.12 \\
\hline $10^{14}$ & & & & & & -90.10 & 18.25 & 33.84 & 43.12 & 52.12 \\
\hline $10^{15}$ & & & & & & -221.98 & 6.60 & 33.32 & 43.10 & 52.12 \\
\hline
\end{tabular}

Table 1: The entries are $\log _{10}(\mathcal{Q})$ for the process $(1)(T+T \rightarrow \mathcal{G})$. The rows are labeled by the density $\rho$ in $\mathrm{gm} / \mathrm{cm}^{3}$, and the columns by the temperature $T$ in $\mathrm{eV}$. A blank entry indicates that $\mathcal{Q}<10^{-320} \mathrm{erg} / \mathrm{cm}^{3}$-s. The number of compact dimensions is $n=2$. 


\begin{tabular}{|c|c|c|c|c|c|c|c|c|c|c|}
\hline$\rho \backslash T$ & 1 & $10^{1}$ & $10^{2}$ & $10^{3}$ & $10^{4}$ & $10^{5}$ & $10^{6}$ & $10^{7}$ & $10^{8}$ & $10^{9}$ \\
\hline 1 & -46.65 & -20.73 & -14.13 & -8.11 & -2.11 & 9.39 & 20.89 & 30.05 & 39.06 & 48.07 \\
\hline $10^{1}$ & -98.74 & -22.73 & -12.70 & -6.61 & -0.61 & 9.39 & 20.89 & 30.05 & 39.06 & 48.07 \\
\hline $10^{2}$ & -267.31 & -37.64 & -11.73 & -5.13 & 0.89 & 9.39 & 20.89 & 30.05 & 39.06 & 48.07 \\
\hline $10^{3}$ & & -89.55 & -13.73 & -3.70 & 2.38 & 9.40 & 20.89 & 30.05 & 39.06 & 48.07 \\
\hline $10^{4}$ & & -255.62 & -28.40 & -2.74 & 3.86 & 9.87 & 20.89 & 30.05 & 39.06 & 48.07 \\
\hline $10^{5}$ & & & -77.05 & -4.58 & 5.27 & 11.28 & 20.89 & 30.05 & 39.06 & 48.07 \\
\hline $10^{6}$ & & & -209.73 & -16.14 & 6.26 & 12.75 & 20.89 & 30.05 & 39.06 & 48.07 \\
\hline $10^{7}$ & & & & -44.73 & 5.46 & 14.04 & 20.90 & 30.05 & 39.06 & 48.07 \\
\hline $10^{8}$ & & & & -106.47 & 0.94 & 14.99 & 21.17 & 30.05 & 39.06 & 48.07 \\
\hline $10^{9}$ & & & & -239.05 & -10.84 & 15.30 & 22.25 & 30.05 & 39.06 & 48.07 \\
\hline $10^{10}$ & & & & & -38.17 & 14.21 & 23.26 & 30.05 & 39.06 & 48.07 \\
\hline $10^{11}$ & & & & & -98.73 & 9.81 & 24.03 & 30.28 & 39.06 & 48.07 \\
\hline $10^{12}$ & & & & & -230.65 & -1.90 & 24.29 & 31.29 & 39.06 & 48.07 \\
\hline $10^{13}$ & & & & & & -29.19 & 23.20 & 32.27 & 39.06 & 48.07 \\
\hline $10^{14}$ & & & & & & -89.74 & 18.81 & 33.03 & 39.29 & 48.07 \\
\hline $10^{15}$ & & & & & & -221.65 & 7.10 & 33.29 & 40.31 & 48.07 \\
\hline
\end{tabular}

Table 2: The entries are $\log _{10}(\mathcal{Q})$ for the process $(2)(T+L \rightarrow \mathcal{G})$. The rows are labeled by the density $\rho$ in $\mathrm{gm} / \mathrm{cm}^{3}$, and the columns by the temperature $T$ in $\mathrm{eV}$. The number of compact dimensions is $n=2$.

\begin{tabular}{|c|c|c|c|c|c|c|c|c|c|c|}
\hline$\rho \backslash T$ & 1 & $10^{1}$ & $10^{2}$ & $10^{3}$ & $10^{4}$ & $10^{5}$ & $10^{6}$ & $10^{7}$ & $10^{8}$ & $10^{9}$ \\
\hline 1 & & -20.43 & -16.94 & -15.08 & -14.01 & 1.16 & 15.49 & 24.75 & 33.78 & 42.81 \\
\hline $10^{1}$ & & -20.91 & -13.70 & -11.58 & -9.96 & 1.16 & 15.49 & 24.75 & 33.78 & 42.81 \\
\hline $10^{2}$ & & & -11.44 & -8.09 & -6.41 & 1.16 & 15.49 & 24.75 & 33.78 & 42.81 \\
\hline $10^{3}$ & & & -11.92 & -4.84 & -2.93 & 1.18 & 15.49 & 24.75 & 33.78 & 42.81 \\
\hline $10^{4}$ & & & & -2.58 & 0.46 & 2.29 & 15.49 & 24.75 & 33.78 & 42.81 \\
\hline $10^{5}$ & & & & -3.15 & 3.31 & 5.47 & 15.49 & 24.75 & 33.78 & 42.81 \\
\hline $10^{6}$ & & & & & 5.33 & 8.82 & 15.49 & 24.75 & 33.78 & 42.81 \\
\hline $10^{7}$ & & & & & 4.84 & 10.97 & 15.49 & 24.75 & 33.78 & 42.81 \\
\hline $10^{8}$ & & & & & & 12.64 & 16.10 & 24.75 & 33.78 & 42.81 \\
\hline $10^{9}$ & & & & & & 13.26 & 18.09 & 24.75 & 33.78 & 42.81 \\
\hline $10^{10}$ & & & & & & & 19.99 & 24.74 & 33.78 & 42.81 \\
\hline $10^{11}$ & & & & & & & 21.36 & 25.18 & 33.78 & 42.81 \\
\hline $10^{12}$ & & & & & & & 19.86 & 27.14 & 33.78 & 42.81 \\
\hline $10^{13}$ & & & & & & & & 28.88 & 33.77 & 42.81 \\
\hline $10^{14}$ & & & & & & & & 29.94 & 34.23 & 42.81 \\
\hline $10^{15}$ & & & & & & & & 27.24 & 36.13 & 42.81 \\
\hline
\end{tabular}

Table 3: The entries are $\log _{10}(\mathcal{Q})$ for the process $(4)(T \rightarrow L+\mathcal{G})$. The rows are labeled by the density $\rho$ in $\mathrm{gm} / \mathrm{cm}^{3}$, and the columns by the temperature $T$ in $\mathrm{eV}$. The number of compact dimensions is $n=2$. 


\begin{tabular}{|c|c|c|c|c|c|c|c|c|c|c|}
\hline$\rho \backslash T$ & 1 & $10^{1}$ & $10^{2}$ & $10^{3}$ & $10^{4}$ & $10^{5}$ & $10^{6}$ & $10^{7}$ & $10^{8}$ & $10^{9}$ \\
\hline 1 & -47.16 & -21.95 & -18.57 & -16.46 & -14.47 & 0.37 & 15.21 & 24.59 & 33.63 & 42.68 \\
\hline $10^{1}$ & -99.50 & -22.88 & -15.35 & -12.99 & -10.97 & 0.37 & 15.21 & 24.59 & 33.63 & 42.68 \\
\hline $10^{2}$ & -268.17 & -38.15 & -12.95 & -9.57 & -7.48 & 0.37 & 15.21 & 24.59 & 33.63 & 42.68 \\
\hline $10^{3}$ & & -90.32 & -13.88 & -6.35 & -4.01 & 0.39 & 15.21 & 24.59 & 33.63 & 42.68 \\
\hline $10^{4}$ & & -256.47 & -28.92 & -3.96 & -0.59 & 1.49 & 15.21 & 24.59 & 33.63 & 42.68 \\
\hline $10^{5}$ & & & -77.82 & -4.80 & 2.58 & 4.76 & 15.21 & 24.59 & 33.63 & 42.68 \\
\hline $10^{6}$ & & & -210.59 & -16.71 & 4.96 & 8.16 & 15.21 & 24.59 & 33.63 & 42.68 \\
\hline $10^{7}$ & & & & -45.50 & 4.83 & 11.09 & 15.22 & 24.59 & 33.63 & 42.68 \\
\hline $10^{8}$ & & & & -107.32 & 0.32 & 13.25 & 15.85 & 24.59 & 33.63 & 42.68 \\
\hline $10^{9}$ & & & & -239.92 & -11.54 & 14.26 & 18.34 & 24.59 & 33.63 & 42.68 \\
\hline $10^{10}$ & & & & & -38.96 & 13.49 & 20.62 & 24.59 & 33.63 & 42.68 \\
\hline $10^{11}$ & & & & & -99.58 & 9.18 & 22.35 & 25.12 & 33.63 & 42.68 \\
\hline $10^{12}$ & & & & & -231.52 & -2.60 & 23.25 & 27.45 & 33.63 & 42.68 \\
\hline $10^{13}$ & & & & & & -29.98 & 22.49 & 29.65 & 33.63 & 42.68 \\
\hline $10^{14}$ & & & & & & -90.59 & 18.18 & 31.36 & 34.17 & 42.68 \\
\hline $10^{15}$ & & & & & & -222.53 & 6.40 & 32.25 & 36.49 & 42.68 \\
\hline
\end{tabular}

Table 4: The entries are $\log _{10}(\mathcal{Q})$ for the process $(3)(L+L \rightarrow \mathcal{G})$. The rows are labeled by the density $\rho$ in $\mathrm{gm} / \mathrm{cm}^{3}$, and the columns by the temperature $T$ in $\mathrm{eV}$. The number of compact dimensions is $n=2$.

\begin{tabular}{|c|c|c|c|c|c|c|c|c|c|c|}
\hline$\rho \backslash T$ & 1 & $10^{1}$ & $10^{2}$ & $10^{3}$ & $10^{4}$ & $10^{5}$ & $10^{6}$ & $10^{7}$ & $10^{8}$ & $10^{9}$ \\
\hline 1 & -46.47 & -20.07 & -10.89 & -1.88 & 7.12 & 16.12 & 25.12 & 34.12 & 43.12 & 52.12 \\
\hline $10^{1}$ & -98.55 & -20.90 & -10.96 & -1.88 & 7.12 & 16.12 & 25.12 & 34.12 & 43.12 & 52.12 \\
\hline $10^{2}$ & -267.11 & -37.46 & -11.07 & -1.89 & 7.12 & 16.12 & 25.12 & 34.12 & 43.12 & 52.12 \\
\hline $10^{3}$ & & -89.37 & -11.91 & -1.96 & 7.12 & 16.12 & 25.12 & 34.12 & 43.12 & 52.12 \\
\hline $10^{4}$ & & -255.41 & -28.22 & -2.12 & 7.11 & 16.12 & 25.12 & 34.12 & 43.12 & 52.12 \\
\hline $10^{5}$ & & & -76.86 & -3.12 & 7.04 & 16.12 & 25.12 & 34.12 & 43.12 & 52.12 \\
\hline $10^{6}$ & & & -209.53 & -15.96 & 6.77 & 16.12 & 25.12 & 34.12 & 43.12 & 52.12 \\
\hline $10^{7}$ & & & & -44.54 & 5.72 & 16.06 & 25.12 & 34.12 & 43.12 & 52.12 \\
\hline $10^{8}$ & & & & -106.27 & 1.11 & 15.91 & 25.12 & 34.12 & 43.12 & 52.12 \\
\hline $10^{9}$ & & & & -238.84 & -10.66 & 15.63 & 25.10 & 34.12 & 43.12 & 52.12 \\
\hline $10^{10}$ & & & & & -37.98 & 14.40 & 25.04 & 34.12 & 43.12 & 52.12 \\
\hline $10^{11}$ & & & & & -98.53 & 9.99 & 24.90 & 34.12 & 43.12 & 52.12 \\
\hline $10^{12}$ & & & & & -230.44 & -1.72 & 24.63 & 34.10 & 43.12 & 52.12 \\
\hline $10^{13}$ & & & & & & -25.04 & 23.40 & 34.04 & 43.12 & 52.12 \\
\hline $10^{14}$ & & & & & & -89.54 & 18.99 & 33.90 & 43.12 & 52.12 \\
\hline $10^{15}$ & & & & & & -221.45 & 7.28 & 33.63 & 43.10 & 52.12 \\
\hline
\end{tabular}

Table 5: The entries are $\log _{10}(\mathcal{Q})$ for the sum of processes (1)-(4). The rows are labeled by the density $\rho$ in $\mathrm{gm} / \mathrm{cm}^{3}$, and the columns by the temperature $T$ in $\mathrm{eV}$. The number of compact dimensions is $n=2$. 


\begin{tabular}{|c|c|c|c|c|c|c|c|c|c|c|}
\hline$\rho \backslash T$ & 1 & $10^{1}$ & $10^{2}$ & $10^{3}$ & $10^{4}$ & $10^{5}$ & $10^{6}$ & $10^{7}$ & $10^{8}$ & $10^{9}$ \\
\hline 1 & 2 & 3 & 1 & 1 & 1 & 1 & 1 & 1 & 1 & 1 \\
\hline $10^{1}$ & 2 & 3 & 1 & 1 & 1 & 1 & 1 & 1 & 1 & 1 \\
\hline $10^{2}$ & 2 & 2 & 3 & 1 & 1 & 1 & 1 & 1 & 1 & 1 \\
\hline $10^{3}$ & 0 & 2 & 3 & 1 & 1 & 1 & 1 & 1 & 1 & 1 \\
\hline $10^{4}$ & 0 & 2 & 2 & 1 & 1 & 1 & 1 & 1 & 1 & 1 \\
\hline $10^{5}$ & 0 & 0 & 2 & 3 & 1 & 1 & 1 & 1 & 1 & 1 \\
\hline $10^{6}$ & 0 & 0 & 2 & 2 & 1 & 1 & 1 & 1 & 1 & 1 \\
\hline $10^{7}$ & 0 & 0 & 0 & 2 & 2 & 1 & 1 & 1 & 1 & 1 \\
\hline $10^{8}$ & 0 & 0 & 0 & 2 & 2 & 1 & 1 & 1 & 1 & 1 \\
\hline $10^{9}$ & 0 & 0 & 0 & 2 & 2 & 1 & 1 & 1 & 1 & 1 \\
\hline $10^{10}$ & 0 & 0 & 0 & 0 & 2 & 2 & 1 & 1 & 1 & 1 \\
\hline $10^{11}$ & 0 & 0 & 0 & 0 & 2 & 2 & 1 & 1 & 1 & 1 \\
\hline $10^{12}$ & 0 & 0 & 0 & 0 & 2 & 2 & 1 & 1 & 1 & 1 \\
\hline $10^{13}$ & 0 & 0 & 0 & 0 & 0 & 3 & 2 & 1 & 1 & 1 \\
\hline $10^{14}$ & 0 & 0 & 0 & 0 & 0 & 2 & 2 & 1 & 1 & 1 \\
\hline $10^{15}$ & 0 & 0 & 0 & 0 & 0 & 2 & 2 & 1 & 1 & 1 \\
\hline
\end{tabular}

Table 6: The entries denote the the process which makes the dominant contribution to $\mathcal{Q}$. A zero indicates that all of the processes are negligible $\left(\mathcal{Q}<10^{-320} \mathrm{erg} / \mathrm{cm}^{3}-\mathrm{s}\right)$.

\begin{tabular}{|c|c|c|c|c|c|c|c|c|c|c|}
\hline$\rho \backslash T$ & 1 & $10^{1}$ & $10^{2}$ & $10^{3}$ & $10^{4}$ & $10^{5}$ & $10^{6}$ & $10^{7}$ & $10^{8}$ & $10^{9}$ \\
\hline 1 & 0.66 & 0.43 & 1.00 & 1.00 & 1.00 & 1.00 & 1.00 & 1.00 & 1.00 & 1.00 \\
\hline $10^{1}$ & 0.65 & 0.97 & 0.98 & 1.00 & 1.00 & 1.00 & 1.00 & 1.00 & 1.00 & 1.00 \\
\hline $10^{2}$ & 0.63 & 0.66 & 0.43 & 1.00 & 1.00 & 1.00 & 1.00 & 1.00 & 1.00 & 1.00 \\
\hline $10^{3}$ & 0.00 & 0.65 & 0.97 & 0.98 & 1.00 & 1.00 & 1.00 & 1.00 & 1.00 & 1.00 \\
\hline $10^{4}$ & 0.00 & 0.63 & 0.66 & 0.39 & 1.00 & 1.00 & 1.00 & 1.00 & 1.00 & 1.00 \\
\hline $10^{5}$ & 0.00 & 0.00 & 0.65 & 0.94 & 0.98 & 1.00 & 1.00 & 1.00 & 1.00 & 1.00 \\
\hline $10^{6}$ & 0.00 & 0.00 & 0.63 & 0.67 & 0.64 & 1.00 & 1.00 & 1.00 & 1.00 & 1.00 \\
\hline $10^{7}$ & 0.00 & 0.00 & 0.00 & 0.65 & 0.56 & 0.99 & 1.00 & 1.00 & 1.00 & 1.00 \\
\hline $10^{8}$ & 0.00 & 0.00 & 0.00 & 0.63 & 0.66 & 0.88 & 1.00 & 1.00 & 1.00 & 1.00 \\
\hline $10^{9}$ & 0.00 & 0.00 & 0.00 & 0.62 & 0.66 & 0.49 & 1.00 & 1.00 & 1.00 & 1.00 \\
\hline $10^{10}$ & 0.00 & 0.00 & 0.00 & 0.00 & 0.65 & 0.64 & 0.98 & 1.00 & 1.00 & 1.00 \\
\hline $10^{11}$ & 0.00 & 0.00 & 0.00 & 0.00 & 0.63 & 0.66 & 0.86 & 1.00 & 1.00 & 1.00 \\
\hline $10^{12}$ & 0.00 & 0.00 & 0.00 & 0.00 & 0.62 & 0.66 & 0.49 & 1.00 & 1.00 & 1.00 \\
\hline $10^{13}$ & 0.00 & 0.00 & 0.00 & 0.00 & 0.00 & 1.00 & 0.64 & 0.98 & 1.00 & 1.00 \\
\hline $10^{14}$ & 0.00 & 0.00 & 0.00 & 0.00 & 0.00 & 0.63 & 0.66 & 0.86 & 1.00 & 1.00 \\
\hline $10^{15}$ & 0.00 & 0.00 & 0.00 & 0.00 & 0.00 & 0.62 & 0.66 & 0.49 & 1.00 & 1.00 \\
\hline
\end{tabular}

Table 7: The entries are the fraction of the contribution to $\mathcal{Q}$ due to the dominant process of Table 6 


\begin{tabular}{|c|c|c|c|c|c|c|c|c|c|c|}
\hline$\rho \backslash T$ & 1 & $10^{1}$ & $10^{2}$ & $10^{3}$ & $10^{4}$ & $10^{5}$ & $10^{6}$ & $10^{7}$ & $10^{8}$ & $10^{9}$ \\
\hline 1 & -56.71 & -30.35 & -20.05 & -10.04 & -0.04 & 9.96 & 19.96 & 29.95 & 39.95 & 49.95 \\
\hline $10^{1}$ & -108.29 & -31.47 & -20.11 & -10.04 & -0.04 & 9.96 & 19.96 & 29.95 & 39.95 & 49.95 \\
\hline $10^{2}$ & -276.35 & -46.70 & -20.36 & -10.05 & -0.04 & 9.96 & 19.96 & 29.95 & 39.95 & 49.95 \\
\hline $10^{3}$ & & -98.11 & -21.48 & -10.10 & -0.04 & 9.96 & 19.96 & 29.95 & 39.95 & 49.95 \\
\hline $10^{4}$ & & -263.66 & -36.47 & -10.36 & -0.05 & 9.96 & 19.96 & 29.95 & 39.95 & 49.95 \\
\hline $10^{5}$ & & & -84.62 & -11.64 & -0.10 & 9.96 & 19.96 & 29.95 & 39.95 & 49.95 \\
\hline $10^{6}$ & & & -216.84 & -23.28 & -0.35 & 9.95 & 19.96 & 29.95 & 39.95 & 49.95 \\
\hline $10^{7}$ & & & & -51.47 & -1.22 & 9.91 & 19.96 & 29.95 & 39.95 & 49.95 \\
\hline $10^{8}$ & & & & -112.85 & -5.45 & 9.78 & 19.95 & 29.95 & 39.95 & 49.95 \\
\hline $10^{9}$ & & & & -245.09 & -16.90 & 9.56 & 19.94 & 29.95 & 39.95 & 49.95 \\
\hline $10^{10}$ & & & & & -43.88 & 8.54 & 19.89 & 29.95 & 39.95 & 49.95 \\
\hline $10^{11}$ & & & & & -104.10 & 4.43 & 19.77 & 29.95 & 39.95 & 49.95 \\
\hline $10^{12}$ & & & & & -235.68 & -6.96 & 19.55 & 29.94 & 39.95 & 49.95 \\
\hline $10^{13}$ & & & & & & -33.90 & 18.54 & 29.89 & 39.95 & 49.95 \\
\hline $10^{14}$ & & & & & & -94.12 & 14.43 & 29.77 & 39.95 & 49.95 \\
\hline $10^{15}$ & & & & & & -225.69 & 3.04 & 29.55 & 39.94 & 49.95 \\
\hline
\end{tabular}

Table 8: The entries are $\log _{10}(\mathcal{Q})$ for the sum of processes (1)-(4) in the case of $n=3$ compact dimensions. The rows are labeled by the density $\rho$ in $\mathrm{gm} / \mathrm{cm}^{3}$, and the columns by the temperature $T$ in $\mathrm{eV}$.

\begin{tabular}{|c|c|c|c|c|c|c|c|c|c|c|}
\hline$\rho \backslash T$ & 1 & $10^{1}$ & $10^{2}$ & $10^{3}$ & $10^{4}$ & $10^{5}$ & $10^{6}$ & $10^{7}$ & $10^{8}$ & $10^{9}$ \\
\hline 1 & -66.96 & -40.44 & -29.15 & -18.15 & -7.15 & 3.85 & 14.85 & 25.85 & 36.85 & 47.85 \\
\hline $10^{1}$ & -118.03 & -41.77 & -29.20 & -18.15 & -7.15 & 3.85 & 14.85 & 25.85 & 36.85 & 47.85 \\
\hline $10^{2}$ & -285.59 & -55.95 & -29.44 & -18.15 & -7.15 & 3.85 & 14.85 & 25.85 & 36.85 & 47.85 \\
\hline $10^{3}$ & & -106.85 & -30.77 & -18.20 & -7.15 & 3.85 & 14.85 & 25.85 & 36.85 & 47.85 \\
\hline $10^{4}$ & & -271.91 & -44.72 & -18.44 & -7.15 & 3.85 & 14.85 & 25.85 & 36.85 & 47.85 \\
\hline $10^{5}$ & & & -92.38 & -19.79 & -7.19 & 3.85 & 14.85 & 25.85 & 36.85 & 47.85 \\
\hline $10^{6}$ & & & -224.16 & -30.59 & -7.41 & 3.85 & 14.85 & 25.85 & 36.85 & 47.85 \\
\hline $10^{7}$ & & & & -58.40 & -8.11 & 3.82 & 14.85 & 25.85 & 36.85 & 47.85 \\
\hline $10^{8}$ & & & & -119.43 & -12.02 & 3.70 & 14.85 & 25.85 & 36.85 & 47.85 \\
\hline $10^{9}$ & & & & -251.33 & -23.14 & 3.51 & 14.84 & 25.85 & 36.85 & 47.85 \\
\hline $10^{10}$ & & & & & -49.79 & 2.69 & 14.80 & 25.85 & 36.85 & 47.85 \\
\hline $10^{11}$ & & & & & -109.68 & -1.13 & 14.69 & 25.85 & 36.85 & 47.85 \\
\hline $10^{12}$ & & & & & -240.93 & -12.19 & 14.51 & 25.83 & 36.85 & 47.85 \\
\hline $10^{13}$ & & & & & & -38.82 & 13.69 & 25.79 & 36.85 & 47.85 \\
\hline $10^{14}$ & & & & & & -98.69 & 9.87 & 25.69 & 36.85 & 47.85 \\
\hline $10^{15}$ & & & & & & -229.93 & -1.19 & 25.51 & 36.83 & 47.85 \\
\hline
\end{tabular}

Table 9: The entries $\operatorname{are~} \log _{10}(\mathcal{Q})$ for the sum of processes (1)-(4) in the case of $n=4$ compact dimensions. The rows are labeled by the density $\rho$ in $\mathrm{gm} / \mathrm{cm}^{3}$, and the columns by the temperature $T$ in $\mathrm{eV}$. 


\begin{tabular}{|c|c|c|c|c|c|c|c|c|c|c|}
\hline$\rho \backslash T$ & 1 & $10^{1}$ & $10^{2}$ & $10^{3}$ & $10^{4}$ & $10^{5}$ & $10^{6}$ & $10^{7}$ & $10^{8}$ & $10^{9}$ \\
\hline 1 & 0.51 & 0.51 & 0.51 & 0.50 & 0.42 & $1.8 \times 10^{-5}$ & $1.4 \times 10^{-8}$ & $1.4 \times 10^{-10}$ & $1.4 \times 10^{-12}$ & $2.8 \times 10^{-14}$ \\
\hline $10^{1}$ & 0.51 & 0.51 & 0.51 & 0.51 & 0.44 & $1.8 \times 10^{-4}$ & $1.4 \times 10^{-7}$ & $1.4 \times 10^{-9}$ & $1.4 \times 10^{-11}$ & $1.4 \times 10^{-13}$ \\
\hline $10^{2}$ & 0.51 & 0.51 & 0.51 & 0.51 & 0.46 & $1.8 \times 10^{-3}$ & $1.4 \times 10^{-6}$ & $1.4 \times 10^{-8}$ & $1.4 \times 10^{-10}$ & $1.4 \times 10^{-12}$ \\
\hline $10^{3}$ & 0.51 & 0.51 & 0.51 & 0.51 & 0.49 & 0.02 & $1.4 \times 10^{-5}$ & $1.4 \times 10^{-7}$ & $1.4 \times 10^{-9}$ & $1.4 \times 10^{-11}$ \\
\hline $10^{4}$ & 0.52 & 0.52 & 0.52 & 0.52 & 0.51 & 0.14 & $1.4 \times 10^{-4}$ & $1.4 \times 10^{-6}$ & $1.4 \times 10^{-8}$ & $1.4 \times 10^{-10}$ \\
\hline $10^{5}$ & 0.56 & 0.56 & 0.56 & 0.56 & 0.56 & 0.37 & $1.4 \times 10^{-3}$ & $1.4 \times 10^{-5}$ & $1.4 \times 10^{-7}$ & $1.4 \times 10^{-9}$ \\
\hline $10^{6}$ & 0.72 & 0.72 & 0.72 & 0.72 & 0.72 & 0.65 & 0.014 & $1.4 \times 10^{-4}$ & $1.4 \times 10^{-6}$ & $1.4 \times 10^{-8}$ \\
\hline $10^{7}$ & 1.20 & 1.20 & 1.20 & 1.20 & 1.20 & 1.20 & 0.14 & $1.4 \times 10^{-3}$ & $1.4 \times 10^{-5}$ & $1.4 \times 10^{-7}$ \\
\hline $10^{8}$ & 2.40 & 2.40 & 2.40 & 2.40 & 2.40 & 2.40 & 1.20 & 0.014 & $1.4 \times 10^{-4}$ & $1.4 \times 10^{-6}$ \\
\hline $10^{9}$ & 5.20 & 5.20 & 5.20 & 5.20 & 5.20 & 5.20 & 4.50 & 0.14 & $1.4 \times 10^{-3}$ & $1.4 \times 10^{-5}$ \\
\hline $10^{10}$ & 11.0 & 11.0 & 11.0 & 11.0 & 11.0 & 11.0 & 11.0 & 1.40 & 0.014 & $1.4 \times 10^{-4}$ \\
\hline $10^{11}$ & 24.0 & 24.0 & 24.0 & 24.0 & 24.0 & 24.0 & 24.0 & 12.0 & 0.14 & $1.4 \times 10^{-3}$ \\
\hline $10^{12}$ & 51.0 & 51.0 & 51.0 & 51.0 & 51.0 & 51.0 & 51.0 & 45.0 & 1.40 & 0.014 \\
\hline $10^{13}$ & 110.0 & 110.0 & 110.0 & 110.0 & 110.0 & 110.0 & 110.0 & 110.0 & 14.0 & 0.14 \\
\hline $10^{14}$ & 240.0 & 240.0 & 240.0 & 240.0 & 240.0 & 240.0 & 240.0 & 240.0 & 120.0 & 1.40 \\
\hline $10^{15}$ & 510.0 & 510.0 & 510.0 & 510.0 & 510.0 & 510.0 & 510.0 & 510.0 & 450.0 & 14.0 \\
\hline
\end{tabular}

Table 10: The entries are the chemical potential $\mu(\rho, T)$. The rows are labeled by the density $\rho$ in $\mathrm{gm} / \mathrm{cm}^{3}$, and the columns by the temperature $T$ in $\mathrm{eV}$.

\begin{tabular}{|c|c|c|c|c|c|c|c|c|c|c|}
\hline$\rho \backslash T$ & 1 & $10^{1}$ & $10^{2}$ & $10^{3}$ & $10^{4}$ & $10^{5}$ & $10^{6}$ & $10^{7}$ & $10^{8}$ & $10^{9}$ \\
\hline 1 & $2.6 \times 10^{-5}$ & $2.2 \times 10^{-5}$ & $-2.3 \times 10^{-4}$ & $-5.8 \times 10^{-3}$ & -0.09 & -0.51 & -0.51 & -0.51 & -0.51 & -0.51 \\
\hline $10^{1}$ & $1.2 \times 10^{-4}$ & $1.2 \times 10^{-4}$ & $3.6 \times 10^{-5}$ & $-3.5 \times 10^{-3}$ & -0.07 & -0.51 & -0.51 & -0.51 & -0.51 & -0.51 \\
\hline $10^{2}$ & $5.6 \times 10^{-4}$ & $5.6 \times 10^{-4}$ & $5.4 \times 10^{-4}$ & $-1.1 \times 10^{-3}$ & -0.05 & -0.51 & -0.51 & -0.51 & -0.51 & -0.51 \\
\hline $10^{3}$ & $2.6 \times 10^{-3}$ & $2.6 \times 10^{-3}$ & $2.6 \times 10^{-3}$ & $2.2 \times 10^{-3}$ & -0.02 & -0.49 & -0.51 & -0.51 & -0.51 & -0.51 \\
\hline $10^{4}$ & 0.01 & 0.01 & 0.01 & 0.01 & 0.00 & -0.37 & -0.51 & -0.51 & -0.51 & -0.51 \\
\hline $10^{5}$ & 0.05 & 0.05 & 0.05 & 0.05 & 0.05 & -0.14 & -0.51 & -0.51 & -0.51 & -0.51 \\
\hline $10^{6}$ & 0.21 & 0.21 & 0.21 & 0.21 & 0.21 & 0.14 & -0.50 & -0.51 & -0.51 & -0.51 \\
\hline $10^{7}$ & 0.71 & 0.71 & 0.71 & 0.71 & 0.71 & 0.68 & -0.37 & -0.51 & -0.51 & -0.51 \\
\hline $10^{8}$ & 1.90 & 1.90 & 1.90 & 1.90 & 1.90 & 1.90 & 0.72 & -0.50 & -0.51 & -0.51 \\
\hline $10^{9}$ & 4.60 & 4.60 & 4.60 & 4.60 & 4.60 & 4.60 & 4.00 & -0.37 & -0.51 & -0.51 \\
\hline $10^{10}$ & 11.0 & 11.0 & 11.0 & 11.0 & 11.0 & 11.0 & 10.0 & 0.86 & -0.50 & -0.51 \\
\hline $10^{11}$ & 23.0 & 23.0 & 23.0 & 23.0 & 23.0 & 23.0 & 23.0 & 11.0 & -0.37 & -0.51 \\
\hline $10^{12}$ & 51.0 & 51.0 & 51.0 & 51.0 & 51.0 & 51.0 & 51.0 & 44.0 & 0.87 & -0.50 \\
\hline $10^{13}$ & 110.0 & 110.0 & 110.0 & 110.0 & 110.0 & 110.0 & 110.0 & 110.0 & 13.0 & -0.37 \\
\hline $10^{14}$ & 240.0 & 240.0 & 240.0 & 240.0 & 240.0 & 240.0 & 240.0 & 240.0 & 120.0 & 0.87 \\
\hline $10^{15}$ & 510.0 & 510.0 & 510.0 & 510.0 & 510.0 & 510.0 & 510.0 & 510.0 & 450.0 & 13.0 \\
\hline
\end{tabular}

Table 11: The entries are $\tilde{\mu}(\rho, T)$ the difference between the chemical potential $\mu(\rho, T)$ and $m_{e}$. The rows are labeled by the density $\rho$ in $\mathrm{gm} / \mathrm{cm}^{3}$, and the columns by the temperature $T$ in $\mathrm{eV}$. 